The Online Interpretation of Sentence-Internal Same and Distributivity

\title{
The Online Interpretation of Sentence-Internal Same and Distributivity*
}

\author{
Jakub Dotlačil \\ UC Santa Cruz
}

\author{
Adrian Brasoveanu \\ UC Santa Cruz
}

\begin{abstract}
This paper investigates how sentence-internal same is processed with three of its licensors (each, all and the) and two orders: licensor+same, i.e., surface scope and same+licensor, i.e., inverse scope. Our study shows that (i) there is no effect of surface vs inverse scope, which we take as an argument for a model-oriented view of the processing cost of inverse scope, and (ii) all is processed faster than each and the with same, which we take as an argument for a particular semantics of distributive licensors.
\end{abstract}

\section{Introduction}

Languages have lexical means to compare two elements and express identity / difference / similarity between them. English uses adjectives of comparison (AOCs) like same, different and similar for this purpose.

AOCs can have both sentence-external and sentence-internal readings. Sentenceexternal readings are so called because in such cases, AOCs compare an element in the current sentence and an element mentioned in a previous sentence, as exemplified in (1) below.

\section{(1) a. Arnold saw 'Waltz with Bashir'. \\ b. Heloise saw the same movie.}

When AOCs have sentence-internal readings, they make a comparison that is internal to the sentence in which they occur without referring to any previously introduced element. This is exemplified in (2) below.

(2) $\left\{\begin{array}{c}\text { Each student } \\ \text { The students } \\ \text { All the students }\end{array}\right\}$ saw the same movie.

* We want to thank Donka Farkas, Berit Gehrke, Tamara Vardomskaya, three anonymous SALT 22 reviewers, and the audiences of UCSC's S-Circle and SALT 22 for comments and discussion. Jakub Dotlačil was supported by a Rubicon grant from the Netherlands Organization for Scientific Research. Adrian Brasoveanu was supported by an SRG grant from the UCSC Committee on Research. The usual disclaimers apply. 
As Carlson (1987) observed, sentence-internal readings must be licensed by a semantically (but not necessarily morphologically) plural element. For example, if we replace the semantically plural subjects in (2) above with a proper name, the only available reading is the sentence-external one, as shown by the example below.

(3) \#Sue saw the same movie.

This paper investigates how sentence-internal same is processed (i) with 3 of its licensors: EACH, ALL and THE and (ii) in 2 scopes: SURFACE-SCOPE, exemplified in (2) above, and INVERSE-SCOPE, exemplified in (4) below. The study has implications for our understanding of inverse scope processing difficulties, as well as the semantics of same and quantifiers, as discussed in the next sections.

(4) The same student saw $\left\{\begin{array}{c}\text { each movie } \\ \text { the movies } \\ \text { all the movies }\end{array}\right\}$.

\subsection{Previous theories and their predictions}

It is generally assumed that in a sentence that has two scopally interacting quantifiers, one scope interpretation is harder to process (Ioup 1975, Tunstall 1998, Anderson 2004, Filik, Paterson \& Liversedge 2004, Reinhart 2006, Radó \& Bott to app., AnderBois, Brasoveanu \& Henderson 2012, among many others). In this paper we will focus on the observation that inverse-scope interpretations are often harder than surface-scope interpretations. Consider for example the sentence below:

(5) A boy climbed every tree.

The most salient and easiest interpretation for this sentence is one in which a single boy climbed every tree (the surface-scope interpretation), as shown in Anderson (2004). This observation can be explained in two different ways. One approach is to explain the difficulties associated with inverse scope in terms of covert scope operations: inverse scope requires an extra operation (Tunstall 1998, Anderson 2004, Reinhart 2006, among others). For example, to derive the inverse-scope reading for the sentence in (5) above, we could imagine that the direct object quantifier moves covertly as shown in (6) below (or type-shifts covertly, for that matter).

(6) $[\text { every tree }]^{x}\left[\right.$ a boy climbed $\left.\_x\right]$

Alternatively, we could explain inverse-scope processing difficulties in terms of changes to the discourse model structure: inverse scope is harder because it requires revising the already built discourse model structure (Fodor 1982; see also Crain \& Steedman 1985, Altmann \& Steedman 1988). 
The Online Interpretation of Sentence-Internal Same and Distributivity

Consider again how sentence (5) is interpreted online. We first hear / read $A$ boy climbed ..., at which point we add a new entity to our discourse model that is a boy and that stands in the climbing relation to whatever direct object we are about to interpret. Then we hear / read the direct object ... every tree. If we want the direct object quantifier to take wide scope, we need to revise the current discourse structure and introduce a set of boys, each of which is associated with a distinct tree. ${ }^{1}$

The AOC same on its sentence-internal reading enables us to distinguish between these two approaches to inverse scope: sentence-internal same has to be scopally licensed but because of its meaning, no revision of the discourse model structure is necessary when a quantifier takes inverse scope over it. Consider the sentence in (7) below: every movie scopes and distributes over same to license its sentence-internal reading (Carlson 1987 among many others), but the model structure will still contain only one student.

(7) The same student saw every movie.

Thus, same can help us distinguish between the two theories of inverse scope.

In addition, previous semantic theories postulated different meanings for same and different meanings for quantificational NPs (Heim 1985, Carlson 1987, Moltmann 1992, Beck 2000, Barker 2007, Dotlačil 2010, Brasoveanu 2011). However, these theories were solely built on native speakers' intuitions about the acceptability and interpretation of sentences with same. Studying the online interpretation of such sentences brings new finer-grained data that can help decide between these proposals.

Sentence-internal readings of AOCs have been previously studied in the psycholinguistic literature, but there is no study of the online interpretation of sentence-internal same in both surface-scope and inverse-scope contexts and with multiple quantificational licensors. Anderson (2004) studied only sentence-internal different and Dwivedi, Phillips, Einagel \& Baum (2010) examined sentence-internal same and different in a surface-scope context only.

The paper is structured as follows. Section 2 describes the experimental method. Section 3 presents the data analysis of one part of the experimental results (reading times) and the emerging generalizations, and section 4 provides an account of these generalizations. Section 5 presents the data analysis and discussion of the second part of the experimental results (participants' answer times), and section 6 concludes.

1 As presented, this theory seems to predict that the scope of quantifiers should always be first and foremost based on their linear order. Such a simplified viewpoint suffices to understand this paper, but we note that the prediction is more complicated. It is possible that the model structure, including the crucial information about the number of entities, is not incrementally constrained / specified as each individual word is processed, but only when certain semantically coherent 'chunks' / domains are processed. Furthermore, if the speaker signals dependency (for instance, by using a dependent indefinite), the hearer most likely uses that information and leaves the relevant parts of the discourse model unspecified to avoid its subsequent revision. 
Dotlačil \& Brasoveanu

\section{Experimental method}

We used a self-paced reading task to test how easy it is to process sentence-internal same

- with 3 licensors: ALL, EACH and THE

- in 2 scopes: SURFACE-SCOPE (quantifier precedes same) and INVERSE-SCOPE (same precedes quantifier)

for a total of $3 \times 2=6$ conditions. Each condition was tested 8 times, 4 times in sentences most likely judged as true relative to the background scenarios and 4 times in sentences most likely judged as false, for a total of 48 stimuli.

Each stimulus begins by introducing a scenario. After reading the scenario, the subjects moved on to a new screen where they read the target sentence word-byword with all the words initially hidden (dashes were displayed where the words should be) and the SPACE bar revealing the next word and hiding the preceding one (self-paced reading task). All scenario+sentence sequences were followed by the same yes/no question, displayed on a new screen. An example in which the stimulus sentence contains the licensor EACH taking surface scope to license same is provided in (8) below: the scenario is given in (8a), the sentence in (8b) and the follow-up question in (8c). The parallel item that exemplifies inverse scope is provided in (9).

(8) a. To prepare for fieldwork, three researchers - a botanist, a historian and a folklorist - had to learn one of two languages - Italian or Japanese. Last year, the botanist learned Italian, the historian also learned Italian and the folklorist learned Italian too.

b. I think that each researcher preparing for fieldwork learned the same language last year.

c. Am I right to think that?

(9) a. To prepare for fieldwork, two researchers - a botanist and a folklorist - had to learn three languages - Italian, Spanish and Portuguese. Last year, the botanist learned Italian, Spanish and Portuguese. The folklorist learned nothing and had to stay at home.

b. I think that the same researcher preparing for fieldwork learned each language last year.

c. Am I right to think that?

In general, scenarios consisted of 2 sets of entities, e.g., researchers and languages, and a relation between them, e.g., the 'learn' relation. In true scenarios, it was specified that all members of one set of entities were related to only one member in the other set. In false scenarios, it was specified that one member of one set of entities was related to a different entity than the other two members. 
The Online Interpretation of Sentence-Internal Same and Distributivity

There were 129 participants in the experiment, divided into 2 groups. The first group consisted of 75 participants and was presented with: (i) 12 stimuli in surface scope-4 items, for each of which we varied the licensor ALL vs EACH vs THE (for a total of $4 \times 3=12$ stimuli), and (ii) 12 stimuli in inverse scope-the remaining 4 items $\times$ the same 3 licensors. The second group consisted of 54 participants and was presented with the same two sets of 4 items, but in the other scope: inverse scope for the first set of 4 and surface scope for the second set of 4 . In each group, half of the 24 stimuli were created so they would be judged as true and the other half as false (6 true and 6 false surface-scope stimuli and 6 true and 6 false inverse-scope stimuli).

There were 24 test stimuli plus 35 fillers (using other quantifiers or the 3 quantificational licensors without same or same with other kinds of licensors, e.g., NP conjunctions), for a total of 59 stimuli per participant.

The participants completed the experiment online and the order was randomized for each participant subject to the condition that any two test items were separated by at least one filler. Out of the 129 participants, 127 were UCSC undergraduate students completing the experiment for (extra-)credit and 2 were volunteers.

The two data sets (75 and 54 participants) were initially analyzed separately but we observed no differences, so the final analysis is based on the merged data sets. 27 participants were excluded because they had $15 \%$ or more incorrect answers.

All responses $\leq 50 \mathrm{~ms}$ and $\geq 2000 \mathrm{~ms}$ were removed and the remaining observations were log transformed. 5 outlier participants with mean log reading times (RTs) more than 2 standard deviations from the grand mean were also excluded. The number of participants included in the final analysis: 97.

\section{Data analysis and resulting generalizations}

Following Trueswell, Tanenhaus \& Garnsey (1994) among others, we factored out the influence of word length and word position by running a linear mixed-effects regression. The regression had intercept-only random effects for subjects and two fixed effects-word length in characters and word position in the sentence. Word position was cubic-spline interpolated with 1 knot demarcating the first 3 words $I$ think that, common to all the stimuli, from the rest of the sentence. The resulting log reading time (RT) residuals were used for all subsequent analyses.

We considered three different regions of interest for the analysis: $(i)$ the quantifier and the following 2 words, (ii) same and the preceding 4 words and the following 2 words, and finally (iii) reading times for full sentences. We studied the first two regions because they reveal the processing cost of quantifier+same either in inverse scope reading (when looking at the quantifier region) or surface scope reading (when looking at the same-region). Regarding the second region of interest, we also considered the 4 words preceding same to see whether any observable effect is 
really due to same or due to the interaction of quantifiers with some other lexical items, say, the main verb or quantifier restriction. Finally, we looked at summed log RT residuals for full sentences because they have been previously argued to reveal the processing cost of inverse scope (Anderson 2004).

\subsection{Quantifier and the following 2 words}

We first examine the quantifier region, which consists of the quantifier (the critical word) and its spillover. The spillover is the first and the second word following the quantifier in the case of EACH and THE; for ALL, we skip the immediately following definitely article the and take the spillover to be the second and third word-in this way, we match spillovers across licensors.

The regions are boldfaced in the two sets of examples below (we always ignore the first part of the target sentence I think that, which always preceded the clause of interest). Note that the spillover has identical words even though the distance from the quantifier is not matched: the words immediately follow the quantifier for $\mathrm{EACH}$ and THE, but they are separated from the quantifier by the in the case of ALL. We return to this issue below.

(10) SURFACE-SCOPE:

a. ... each researcher preparing for fieldwork learned the same language last year.

b. ...the researchers preparing for fieldwork learned the same language last year.

c. ....all the researchers preparing for fieldwork learned the same language last year.

(11) INVERSE-SCOPE:

a. ... the same researcher preparing for fieldwork learned each language last year.

b. ...the same researcher preparing for fieldwork learned the languages last year.

c. ...the same researcher preparing for fieldwork learned all the languages last year.

We study the region word by word and we analyze the data in a linear mixedeffects regression. The regression has three fixed effects related to our experimental manipulations: quantifier type (ALL vs EACH vs THE), scope (SURFACE-SCOPE vs INVERSE-SCOPE) and the interaction of scope and quantifier type.

As we mentioned above, there are irrelevant differences between ALL on one hand and EACH and THE on the other hand. Consider, for example, the spillover 


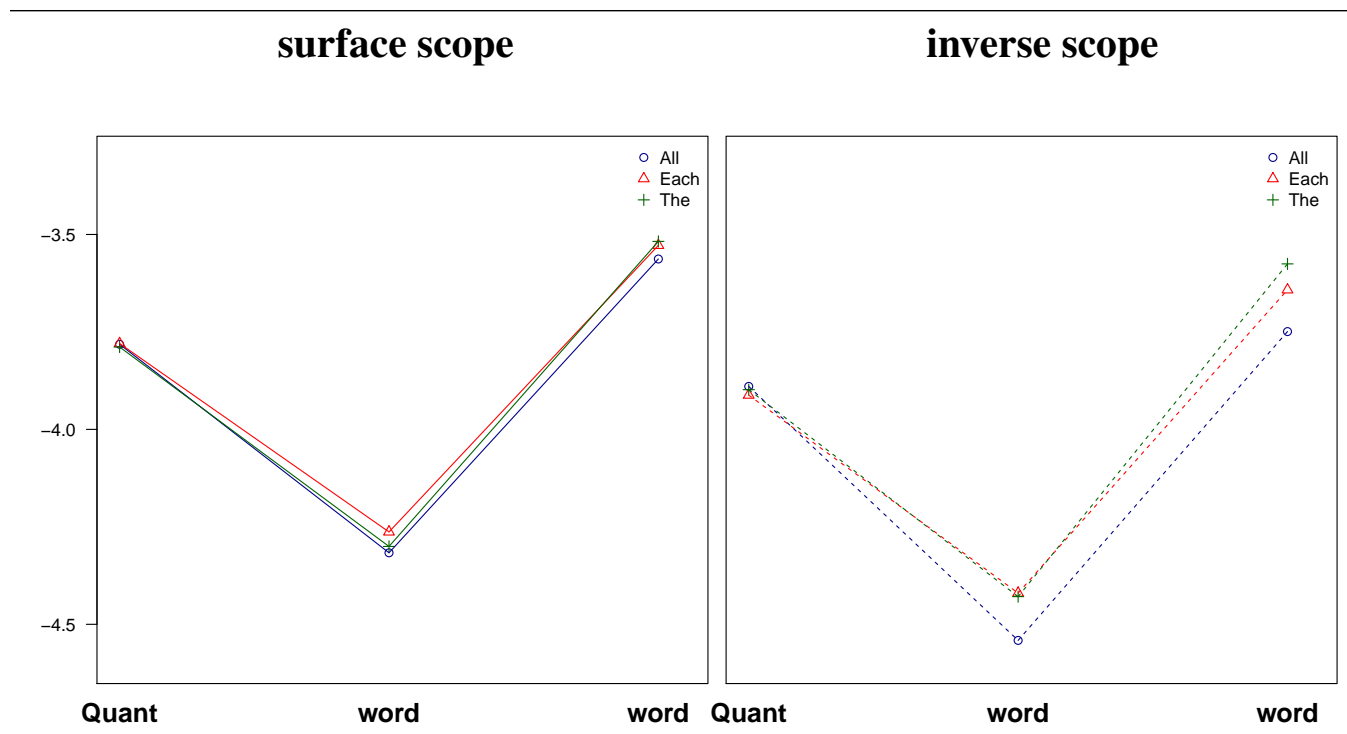

Figure 1 Effects of quantifier and scope on residual log RTs for quantifier plus spillover

language(s) in (11). This is immediately preceded by the quantifier in first two sentences but it is separated from the quantifier by the in the last case. Furthermore, the verb is only two words away from the spillover in the first two sentences, but it is three words away in case of ALL. To control for these differences in our analysis, we included $\log$ RTs of the three preceding words as additional fixed-effect predictors in our model (see Vasishth \& Lewis 2006 for discussion and justification). Once the variation induced by the differences in the preceding words / regions is factored out in this way, we can be confident that whatever effects we find are due to the experimental manipulations.

Our model also included intercept-only random effects for subjects and items.

Figure 1 plots the coefficients of the resulting linear mixed-effects regression for each quantifier type and both scopes. The values on the $y$-axis are negative because we analyze residual $\log$ RTs - recall that word length and word position have already been factored out.

We observe that SURFACE-SCOPE takes more time than INVERSE-SCOPE, but the two scopes are not directly comparable for two reasons: (i) the quantifier is in a different syntactic position (subject vs. object) in the two cases, and (ii) the reader has only seen the quantifier in the case of SURFACE-SCOPE, while in the case of INVERSE-SCOPE the reader has seen both the quantifier and same.

We are also not interested in the effect of quantifier type per se. The quantifiers differ in several respects (frequency, length, singular/plural number), all of which are irrelevant for our semantic study.

Thus, the only theoretically important result is the interaction of scope and quantifier 
type, which reveals what extra processing cost is tied to a particular quantifier when the quantifier licenses same. While the interaction is not significant on the critical word, it is significant on both words of the spillover, ${ }^{2}$ where EACH and THE are read more slowly than ALL in inverse scope. ${ }^{3}$ The effect can be also easily observed in Figure 1: consider the difference between EACH and THE on one hand and ALL on the other hand, which is much larger in inverse scope than surface scope. This conclusion is summarized below:

(12) Generalization 1. In inverse scope, EACH and THE cause slower reading times (of the spillover of the quantifier region) than ALL.

Generalization 1 does not simply capture a general difference between the quantifiers EACH, THE and ALL. If it had, the same slowdown would have been visible in the surface scope condition (it is not). So the difference must be due to the interaction of quantifier type (ALL vs EACH/THE) and scope (SURFACE VS INVERSE).

\subsection{Same and the preceding and following words}

We turn now to the same region, which includes the word same, the preceding 4 words and the following 2 words. Once again, the words to be analyzed are boldfaced in the example below. Only surface scope (see (13)) is of interest because same precedes any experimental manipulation in the inverse-scope condition, as illustrated in (14). We will therefore focus exclusively on surface scope for this part of the analysis.

(13) SURFACE-SCOPE:

... each researcher preparing for fieldwork learned the same language last year.

... the researchers preparing for fieldwork learned the same language last year.

... all the researchers preparing for fieldwork learned the same language last year.

(14) INVERSE-SCOPE:

... the same researcher preparing for fieldwork learned each language last year.

2 The likelihood ratio test comparing the current model and the model that lacks the interaction effects is significant at $p<.001\left(\chi^{2}=17.28, \mathrm{df}=2\right)$ for the first word in the spillover. It is also significant at $p<.001\left(\chi^{2}=25.37, \mathrm{df}=2\right)$ for the second word in the spillover.

3 The same results were also obtained when the words in the spillover were not identical but their distance from the quantifier was. That is, we considered the two words immediately following the quantifier ALL, which where the+noun, and compared them to the two words immediately following EACH and THE. 
The Online Interpretation of Sentence-Internal Same and Distributivity

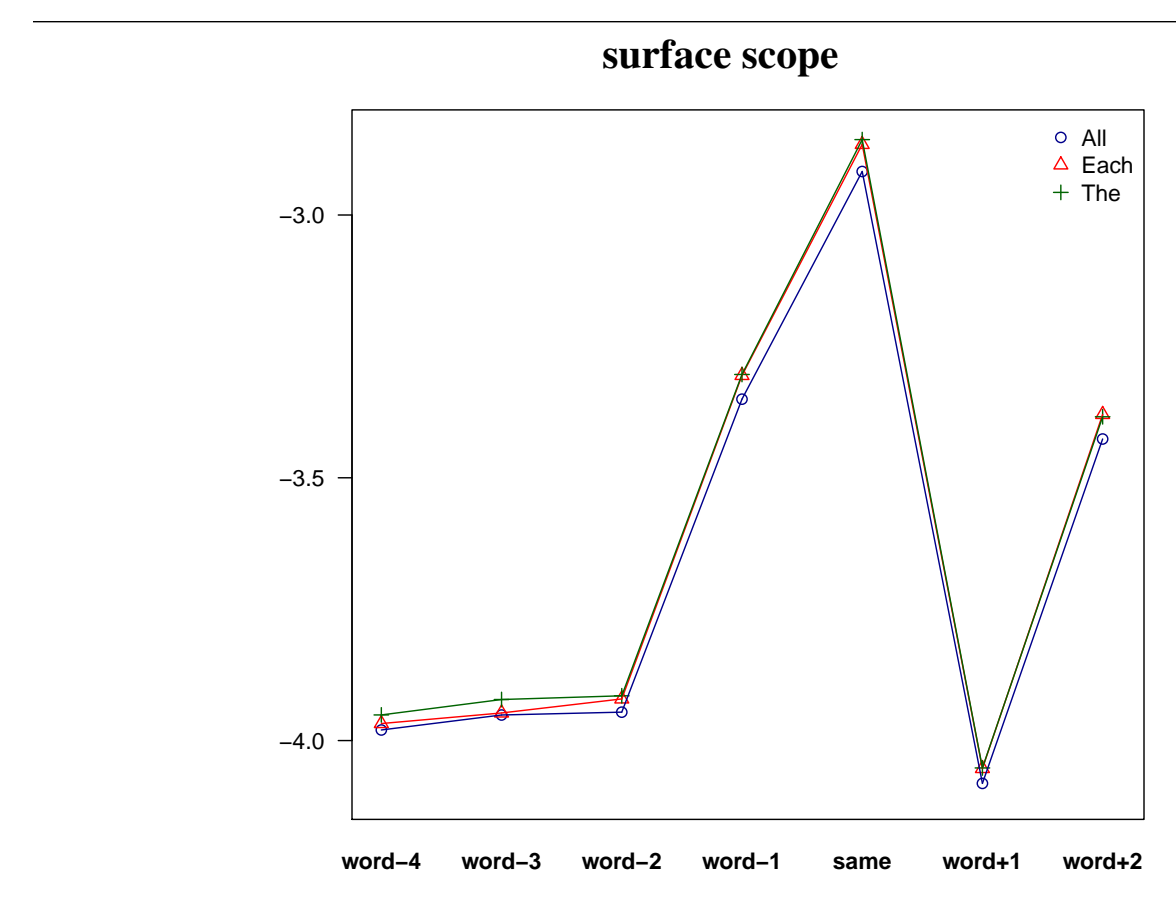

Figure 2 Effects of QUANT and ORD on residual log RTs for same and preceding and following words

The results are plotted in Figure 2. We observe that EACH and THE lead to significant slowdowns in three regions: same, the word preceding same (always the definite article) and the second word in the spillover. ${ }^{4}$ In other regions the effect of quantifier did not reach significance.

The second generalization, provided below, summarizes these results:

(15) Generalization 2. In surface scope, EACH and THE cause slower reading times than ALL when reading same, its preceding word and the spillover. ${ }^{5}$

4 The likelihood ratio test comparing the model which includes the effect of quantifer and the model which lacks the effect is significant at $p<.001\left(\chi^{2}=18.86, \mathrm{df}=2\right)$ on same. It is significant at $p<.01\left(\chi^{2}=12.43, \mathrm{df}=2\right)$ on the word preceding same and significant at $p<.05\left(\chi^{2}=7.23\right.$, $\mathrm{df}=2$ ) on the second word in the spillover.

5 It might be puzzling that we find a significant effect of quantifier type on the second word after same but not on the word immediately following same. This is a consequence of the arbitrary cut-off point of significance tests: EACH and THE are numerically slower in the first word after same as well, but this effect crosses the threshold of signficance only on the second word. Notice also that the effect on that second spillover word is significant only at the level of $p<.05$. 
Dotlačil \& Brasoveanu

\subsection{Reading times for the full sentence}

Finally, we examine the full sentences by summing the residual log RTs. The resulting total times are plotted in Figure 3. We see that both quantifier type and scope are significant factors in predicting reading times for full sentences. ${ }^{6}$

But when we subset the data by quantifier type, we see that there is no significant effect of scope in the case of ALL. In contrast, there is a significant effect of scope in the case of both EACH and THE. ${ }^{7}$ We therefore extract the following generalizations:

(16) Generalization 3. Surface scope and inverse scope take about the same time for ALL.

(17) Generalization 4. Inverse scope takes more time than surface scope for EACH and THE.

(18) Generalization 5. Overall, EACH and THE are slower than ALL.

\section{Analysis}

Our account of these generalizations makes use of three assumptions, already proposed and argued for in the previous literature: $(i)$ sentence-internal same is ambiguous, (ii) the quantifier each requires event differentiation, and (iii) the definite determiner the has three ordered interpretations. Our experimental findings can be seen as providing additional empirical support for these three assumptions, to the detailed presentation of which we now turn.

\subsection{Three assumptions}

Our first assumption is that same is ambiguous, just like different is in many languages (Beck 2000, Dotlačil 2010). This is exemplified below for Dutch (for more discussion, see Dotlačil 2010). The sentence-internal reading of ander, glossed as different [1] in (19) below, is licensed by the distributive quantifier iedere (each) and to a lesser extent by alle (all), but not by the definite de (the). In contrast, the sentence-internal reading of verschillende, glossed as different[2] in (20), is licensed by the definite de and by alle, but not by iedere. Beck (2000) introduces

6 The likelihood ratio test comparing the model with both effects and the model without the quantifier effect is significant at $p<.001\left(\chi^{2}=25.71, \mathrm{df}=2\right)$. The likelihood ratio test comparing the model with both effects and the model without the scope effect is significant at $p<.01\left(\chi^{2}=9.41\right.$, df $\left.=1\right)$.

7 In the case of EACH and THE, the likelihood ratio test comparing the model with SCOPE and the model without SCOPE is significant at $p<.05\left(\chi^{2}=4.96, \mathrm{df}=1\right)$ and $p<.01\left(\chi^{2}=8.44\right.$, df $\left.=1\right)$, respectively. In the case of ALL, the likelihood ratio test clearly fails to reach significance $(p>.1$, $\left.\chi^{2}=.01, \mathrm{df}=1\right)$. 
The Online Interpretation of Sentence-Internal Same and Distributivity
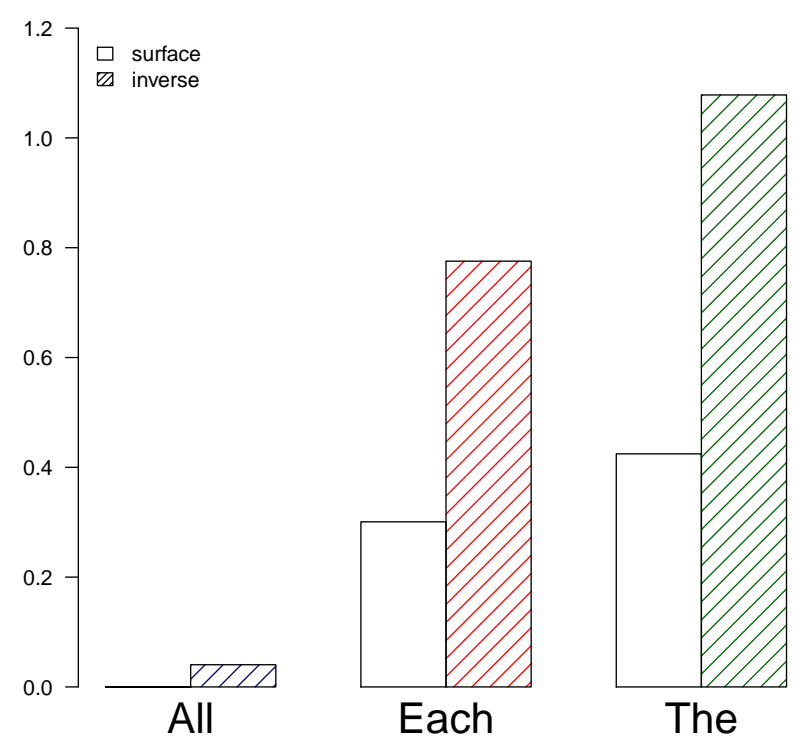

Figure 3 Effects of QUANT and ORD on summed residual log RTs for full sentences

and discusses the same contrast for the German counterparts of these two lexical items.

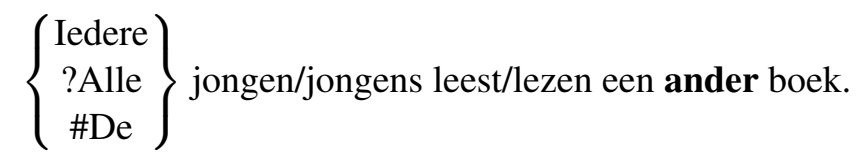

$\left\{\begin{array}{c}\text { Every } \\ \text { ?All } \\ \text { \#The }\end{array}\right\}$ boy/boys reads/read a different $[1]$ book.

(20) $\left\{\begin{array}{c}\text { \#Iedere } \\ \text { Alle } \\ \text { De }\end{array}\right\}$ jongen/jongens leest/lezen verschillende boeken.

$\left\{\begin{array}{c}\text { \#Every } \\ \text { All the } \\ \text { The }\end{array}\right\}$ boy/boys reads/read different[2] books.

We assume that just as different in English is covertly ambiguous between these two meanings, same is ambiguous too. Same[1] simply expresses identity between two entities and can have (i) a sentence-external reading, exemplified in (21) below (repeated from above), or (ii) a sentence-internal reading under the distributive quantifier EACH or under the distributive interpretation of ALL, exemplified in (22). 
(21) a. Arnold saw 'Waltz with Bashir'.

b. Heloise saw the same[1] movie. [the movie seen by Heloise $=$ 'Waltz with Bashir'] $\left\{\begin{array}{c}\text { Each boy } \\ \text { All the boys }\end{array}\right\}$ saw the same[1] movie. [for any two boys $b_{1}$ and $b_{2}, b_{1}$ 's movie $=b_{2}$ 's movie]

The sentence-external reading is straightforward to analyze: same is anaphoric to the movie 'Waltz with Bashir' mentioned in the previous sentence and requires the movie seen by Heloise to be identical to the anaphorically retrieved movie.

We follow Brasoveanu (2011) here and assume that sentence-internal same under distributive quantifiers like $\mathrm{EACH}$ also has this simple denotation that requires the identity of two entities. The extra work needed to derive the sentence-internal reading is performed by the distributive quantifier, which temporarily creates in its scope interpretation contexts of sentence-external form. The basic idea of the analysis is depicted in (23) below: the distributive quantifier EACH takes the set of all boys $\left\{\right.$ boy $_{1}$, boy $_{2}$, boy $\left._{3}\right\}$ contributed by its restrictor and predicates its nuclear scope of all pairs of individual boys. That is, for each two boys, we introduce their corresponding movies and check that they are identical. Thus, same in the scope of distributive quantification has access to temporarily-created interpretation contexts of the same form as the ones needed for its sentence-external reading.

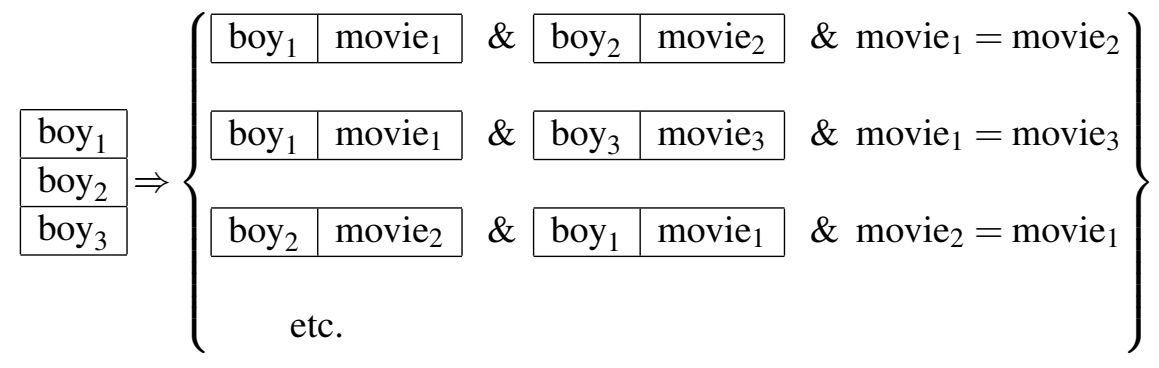

In contrast, same [2] relates parts of a plural individual to one entity by a binary relation $R$, along the lines of the analyses proposed in Dowty (1985) and Barker (2007). Consider (24) below:

(24) $\left\{\begin{array}{c}\text { The boys } \\ \text { All the boys }\end{array}\right\}$ saw the same[2] movie.

The plural individual is the collection of boys and the relation $R$ is 'see movie'. Same [2] requires 'see movie' to relate any two boy-atoms to the same entity, i.e., any two atomic parts of the plural individual are related to the same movie.

Thus, in the case of sentence-internal readings with same[1], all the work is done by the distributive quantifier (the licensor), while in the case of sentence-internal 
The Online Interpretation of Sentence-Internal Same and Distributivity

readings with same [2], all the work is done by same, which needs a non-distributive plurality as the licensor so it can relate its parts to one and the same entity.

Importantly, EACH is only compatible with same[1], THE is only compatible with same [2], while ALL is compatible with both due to its optional distributivity.

Our second assumption is that the distributive quantifier EACH requires event differentiation in its scope, as Tunstall (1998) argues (see also Beghelli \& Stowell 1997). Consider the contrast in example (25) below: there is only one event of taking a photograph, so each is less acceptable than either every or all because the events associated the individual students are not differentiated (they're the same event).

(25) Jake photographed $\left\{\begin{array}{c}\# \text { each student } \\ \text { every student } \\ \text { all the students }\end{array}\right\}$ in the class, but not separately.

Our third and final assumption is that there is a preference ordering over the three possible readings - collective, cumulative and distributive - that the definite determiner THE can have. This ordering is provided in (26) below, based on previous work by Brooks \& Braine (1996), Frazier, Pacht \& Rayner (1999) and Dotlačil \& Brasoveanu (in prep.).

(26) The preferred reading of THE is collective, e.g., (27a) below, followed by a cumulative reading, e.g., (27b), which in turn is followed by a distributive reading, e.g., (27c).

(27) a. The boys elected the representative. (collective)

b. The boys hugged the girls. (cumulative)

c. The boys had a sip of juice. (distributive)

\subsection{Accounting for the five generalizations}

We turn now to the account of the five generalizations listed above.

First, recall that EACH requires event differentiation in its scope (Tunstall 1998). Because of this, we have a very strong preference for distinct languages when we interpret sentence (28) below (Anderson 2004, Roeper, Pearson \& Grace 2011).

(28) Each researcher learned a language.

By the same token, EACH is a dispreferred licensor of sentence-internal same because licensing same - as in (29) below-goes against the default tendency to establish event differentiation in terms of a direct object with varying/dependent reference. 
(29) Each researcher learned the same language.

We consequently expect EACH to take more time than ALL when we look at the word same and its spillover in the surface-scope condition. This is exactly what we discovered (see Generalization 2 in (15) above).

The same reasoning explains why EACH leads to greater full-sentence reading times than ALL (see Generalization 5 in (18) above).

Generalization 2 also showed that there was slowdown on the word preceding same, namely the definite article. There are two possible explanations. First, definites exhibit dependent reference only in special circumstances (see for example Chierchia 1995 among others for a discussion of dependent definites), so the definite article is also at odds with the event differentiation requirement contributed by $\mathrm{EACH}$, just as same is. Alternatively, speakers mights have anticipated same at the point of encountering the since the two coocurred repeatedly in the experiment.

Turning now to THE, we mentioned above that it is interpreted collectively by default. Due to this preference, it is by default incompatible with same since sentence-internal same is not licensed in collective readings:

(30) \#The boys elected the same president.

Reanalyzing THE towards a non-collective reading takes extra time, hence we expect to see THE taking more time than ALL when we look at same and its spillover. Again, this is what we find-see Generalization 2 in (15) above. We repeat the relevant example below for convenience and boldface the relevant measured regions. ${ }^{8}$

8 Once again, the slowdown observable on the definite article preceding same might be due to readers' expectation that same will follow. Interestingly, THE caused slower reading times than ALL in each of the 4 regions preceding same, even though this slowdown was only close to significant in most of these regions. The only region where it was (highly) significant was the definite article immediately preceding same.

Taking this general slowdown as real for a moment, how could we explain it? One possibility is that at least some experimental items required the nominal restrictor of THE (e.g., preparing for fieldwork in (31) above) to be understood distributively given the background scenario, and this is at odds with the preferably collective interpretation of THE. But we did not control for the interpretation of the restrictor in the experimental setup, so not every test item had a distributively interpreted restrictor-and the test items that had this interpretation could require it at different points in the sentence. This might explain the fact that the effect was only close to significant, unlike other effects we discuss in this section. Another possibility is that the effect is in fact spurious since it failed to reach significance even with 97 participants.

We leave the exploration of this issue for future research. Note only that the spillover fixed-effect predictors that were added in the analysis of every region of interest factored out this extra processing load, so the significant effects of THE that we see in our regions of interest occur in addition to this controlled-for processing load. 
The Online Interpretation of Sentence-Internal Same and Distributivity

(31) $\ldots$ \{the vs. all the $\}$ researchers preparing for fieldwork learned the same language last year.

The extra time required to reanalyze THE also explains the fact that THE takes more time than ALL when we look at full-sentence reading times in the surface-scope condition (see Generalization 5 in (18) above).

We see no difference in full-sentence reading times between the inverse-scope and the surface-scope condition for ALL (see Generalization 3 in (16) above). This is expected if the processing cost associated with inverse scope could only arise because of model structure revisions, since no revision takes place in our experiment. Crucially, the result is unexpected if the processing cost of inverse scope was tied to covert scoping operations.

We note that another possible explanation for these findings-namely, that AOCs somehow do not cause processing difficulties when requiring inverse scope-cannot be right given that Anderson (2004) found that sentences with different led to slowdown in inverse-scope conditions. The difference between our results with same and Anderson's results with different is predicted by the hypothesis that model structure revision, not inverse-scope taking, is costly: different, unlike same, leads to changes in discourse model structure when its quantificational licensors are forced to take inverse scope.

In contrast, we observed a slowdown for EACH and THE in the inverse-scope vs the surface-scope condition when we examined full-sentence reading times (see Generalization 4 in (17) above). Attributing this slowdown to covert scoping operations would leave unexplained the lack of contrast between inverse and surface scope for ALL. We therefore attribute it to the fact that EACH and THE force late disambiguation for same: EACH is only compatible with same [1], while THE (on its default reading) is only compatible with same[2] (see Brasoveanu 2011 and Brasoveanu \& Dotlačil 2012 for more detailed studies of the relative acceptability of sentence-internal items with a variety of licensors in English). Importantly, disambiguation is forced late in the sentence since same occurs in subject position, and late disambiguation takes extra time (Clifton \& Staub 2008).

The same explanation in terms of forced late disambiguation of same can be provided for the EACH/THE vs. ALL slowdown observed in the inverse-scope condition on the quantifier spillover (see Generalization 1 in (12) above). Same, which appears in subject position, does not need to be disambiguated when the processor encounters ALL, but it must be disambiguated when the processor encounters EACH or THEand as already noted, late disambiguation induces greater reading time. Once again, the relevant example is repeated below for convenience and the measured regions are boldfaced.

(32) ...the same researcher preparing for fieldwork learned $\left\{\begin{array}{l}\text { each language } \\ \text { the languages }\end{array}\right\}$ last year. 
Dotlačil \& Brasoveanu

\section{Answer times}

We now turn to the second dependent variable that we measured in the experiment: answer time. This is the time it took a participant to answer the question that appeared after the target sentence. The question was always the same (Am I right to think that?), see (8c) and (9c) above. The experiment was set up in such a way that each experimental manipulation (SURFACE/INVERSE $\times$ ALL/EACH/THE) should receive a negative response half the time and a positive response the other half, given the background scenario.

We analyzed answer times as a dependent variable in a linear mixed-effects regression model with 4 fixed effects-QUANTIFIER, SCOPE, CORRECT-ANSWER (i.e., is the answer correct or not?) and POSITIVE-ANSWER (i.e., is the correct answer positive?) - and their 2-way interactions. In addition, the model had subject random effects for the intercept and the CORRECT-ANSWER slope, and item random effects for the intercept only. This model had the best data fit among all models whose estimation converged; adding further predictors did not lead to significant improvements. We took as outliers all the observations whose residuals in this model were more than 2.5 standard deviations away from the mean and we excluded them.

There were four significant effects in this final model:

(i) the inverse scope of the target sentence led to significant slowdown in answer times $(t=2.66, p<.01)$;

(ii) incorrect responses were significantly slower $(t=2.18, p<.05)$;

(iii) incorrect responses led to even more slowdown when the expected answer was positive (interaction of CORRECT-ANSWER and POSITIVE-ANSWER, $t=3.51, p<.001)$;

(iv) inverse-scope stimuli also led to even more slowdown when the expected answer was positive (interaction of CORRECT-ANSWER and SCOPE, $t=4.58, p<$ $.001)$.

Let us start with the effect of incorrect responses. The fact that participants hesitated before answering incorrectly is probably not very surprising. This is most likely due to the fact that participants were systematically less sure about their responses in these cases and hesitated more.

Incorrect responses were even slower when participants (wrongly) selected the negative response, which might be an interesting case of positive bias: it was easier for participants to incorrectly accept a sentence than to incorrectly reject it. This suggests that participants are more hesitant to reject rather than accept a statement when unsure about it.

Moving on to the effect of scope, we observe that inverse-scope sentences caused slower response times in general, and even more so when the response was incorrect. 
One might think that these results finally support the hypothesis that the extra processing cost of inverse scope is due to additional syntactic and/or semantic operations. This hypothesis could in principle be defended by arguing that the lack of inverse-scope slowdown in the self-paced reading part of the task is due either $(i)$ to the fact that the particpants postponed the calculation of scope until answering the question or (ii) to the fact that they always kept the surface scope interpretation, which penalized them when answering the question.

We find this explanation unlikely. It is true that extra processing cost was found in sentences following the introduction of scopal elements (Tunstall 1998). However, this happened when the follow-up sentence disambiguated scopal readings. In our experiment, the question itself (Am I right to think that?) did not disambiguate anything, so it is not clear why readers should reconsider their original interpretation or be penalized for selecting surface scope at this point in the task. Moreover, such a theory would have to account for the different behavior of EACH/THE vs ALL with respect to inverse scope in the self-paced reading part of the task (while accounting for the lack of any quantifier effect in the question part).

In fact, there are good reasons to believe that the calculation of inverse scope had to happen rapidly, in the sentence in which the quantifiers appeared. Recall that we used same in our stimuli, which had to be in the scope of a quantifier so that its sentence-internal reading could be licensed. If this anaphoric requirement had not been satisfied, the sentence would have been infelicitous. Consider for example (33) below, repeated from above. Participants could postpone assigning inverse scope to all the languages only if they thought that the anaphoric requirement contributed by same could be resolved in some other way. But no such alternative resolution was salient in the context.

... the same researcher preparing for fieldwork learned all the languages last year.

Moreover, it is independently known that readers try to resolve anaphoric requirements as quickly as possible, and in fact anticipate that the resolution should take place even before it happens (Kazanina, Lau, Lieberman, Yoshida \& Phillips 2007). The null hypothesis is therefore that this is also true for the interpretation of same: readers resolve the anaphoric requirement of same as soon as possible and assign inverse scope to direct object quantifiers in the process.

The question that remains is why inverse scope seems to lead to increased answer times. We tentatively suggest that this is not due to scope but it is simply a consequence of the extra time needed for memory recall in those cases. When participants read the question Am I right to think that?, they have to recall the original scenario. For example, they have to remember which researcher learned which language. In particular, they have to recall (34a) below for the surface-scope sentence (all the researchers ... the same language) and (34b) for the inverse-scope sentence (the same researcher ... all the languages). 
(34) a. Last year, the botanist learned Italian, the historian also learned Italian and the folklorist learned Italian too.

b. Last year, the botanist learned Italian, Spanish and Portuguese. The folklorist learned nothing and had to stay at home.

The crucial information that has to be recalled is presented differently in (34a) and (34b). In the case of surface scope, the researchers and their associated language are presented one by one. In the case of inverse scope, all the languages are presented together in one clause and are associated with one researcher, and there is an additional clause about the other researcher.

Because of this, the temporal distance between entity presentations for one of the two sets of entities under discussion is always smaller for inverse-scope scenarios-e.g., the three languages in (34b) above receive almost identical time stamps. Given the generally accepted assumption that memories (or at least short-term memories) are encoded with a temporal component (e.g., Lewis \& Vasishth 2005, Brown, Neath \& Chater 2007 and references therein), this makes the recall of inverse scope scenarios more difficult and presumably also more time consuming.

In addition, both scenarios introduced the researchers first and the languages next. This matches the order of presentation in surface but not inverse scope sentences. The different orders of presentation used in inverse scope stimuli (the scenario order vs the sentence order) might have influenced memory encoding and/or storage, which in turn would have an effect on the participants' answer times.

\section{Conclusion}

The paper argued for two main claims, based on a self-paced reading study of sentence-internal same with three quantificational licensors (ALL, EACH and THE).

First, the inverse scope of quantifiers seems to be costly because of model structure reanalysis, not (only) because of covert scope operations. The main observation supporting this was the fact that there is no slowdown when ALL takes inverse scope over same to license its sentence-internal reading. The slowdown observed when EACH and THE take inverse scope is attributed to the fact that they require same to be disambiguated. Since this requirement is contributed late in the sentence (same occurs in subject position and EACH/THE in direct object position), a certain amount of backtracking is necessary and this adds to processing time.

Second, the surface-scope slowdown for EACH/THE relative to ALL was taken as an argument for lexically-specified differences between these distributors, which make EACH and THE less compatible than ALL with sentence-internal same. This result is in line with the corpus study reported in AnderBois et al. (2012), where lexically-specified scoping preferences were argued to be a very strong, if not the strongest, predictor of quantifier scope. 
The Online Interpretation of Sentence-Internal Same and Distributivity

\section{References}

Altmann, Gerry \& Mark Steedman. 1988. Interaction with context during human sentence processing. Cognition 30. 191-238.

AnderBois, Scott, Adrian Brasoveanu \& Robert Henderson. 2012. The pragmatics of quantifier scope: A corpus study. In A. Aguilar-Guevara, A. Chernilovskaya \& R. Nouwen (eds.), Proceedings of Sinn und Bedeutung 16, Cambridge, MA: MIT Working Papers in Linguistics.

Anderson, Catherine. 2004. The structure and real-time comprehension of quantifier scope ambiguity. Evanston, Illinois: Northwestern University dissertation.

Barker, Chris. 2007. Parasitic scope. Linguistics and Philosophy 30. 407-444.

Beck, Sigrid. 2000. The semantics of Different: Comparison operator and relational adjective. Linguistics and Philosphy 23. 101-139.

Beghelli, Filippo \& Timothy Stowell. 1997. Distributivity and negation. In Anna Szabolcsi (ed.), Ways of scope taking, 71-109. Dordrecht: Kluwer.

Brasoveanu, Adrian. 2011. Sentence-internal Different as quantifier-internal anaphora. Linguistics and Philosophy 34. 93-168.

Brasoveanu, Adrian \& Jakub Dotlačil. 2012. Licensing sentence-internal readings in english. In Amsterdam colloquium 2011, 122-132. Heidelberg: Springer.

Brooks, Patricia J. \& Martin D.S. Braine. 1996. What do children know about the universal quantifiers all and each? Cognition 60(3). 235-268.

Brown, G. D. A., I. Neath \& N. Chater. 2007. A temporal ratio model of memory. Psychological Review 114. 539-576.

Carlson, Gregory. 1987. Same and Different: some consequences for syntax and semantics. Linguistics and Philosphy 10. 531-565.

Chierchia, Gennaro. 1995. Dynamics of meaning: Anaphora, presupposition, and the theory of grammar. Chicago: University of Chicago Press.

Clifton, Charles \& Adrian Staub. 2008. Parallelism and competition in syntactic ambiguity resolution. Language and Linguistics Compass 2. 234-250.

Crain, Stephen \& Mark Steedman. 1985. On not being led up the garden path: the use of context by the psychological syntax processor. In L. Karttunen David Dowty \& Arnold Zwicky (eds.), Natural language parsing: Psychological, computational and theoretical perspectives, 320-358. Cambridge: Cambridge University Press.

Dotlačil, Jakub. 2010. Anaphora and distributivity. A study of same, different, reciprocals and others. Utrecht: Utrecht University dissertation.

Dotlačil, Jakub \& Adrian Brasoveanu. in prep. The on-line interpretation of the distributivity and its sources. Ms.

Dowty, David. 1985. A unified indexical analysis of same and different: A response to stump and carlson. Ms., presented at University of Texas Workshop on Syntax and Semantics, Austin, Texas.

Dwivedi, Veena D., Natalie A. Phillips, Stephanie Einagel \& Shari R. Baum. 2010. The neural underpinnings of semantic ambiguity and anaphora. Brain Research 
1311. 93-109.

Filik, Ruth, Kevin B. Paterson \& Simon P. Liversedge. 2004. Processing doubly quantified sentences: Evidence from eye movements. Psychonomic Bulletin \& Review 11(5). 953-959.

Fodor, Janet Dean. 1982. The mental representation of quantifiers. In Stanley Peters \& Esa Saarinen (eds.), Processes, beliefs and questions, 129-164. Dordrecht: Reidel.

Frazier, Lyn, Jeremy M. Pacht \& Keith Rayner. 1999. Taking on semantic commitments, II: collective versus distributive readings. Cognition 70. 87-104.

Heim, Irene. 1985. Notes on comparatives and related matters. Ms.

Ioup, Georgette. 1975. Some universals for quantifier scope. In J. Kimball (ed.), Syntax and semantics 4, 37-58. New York: Academic Press.

Kazanina, Nina, Ellen F. Lau, Moti Lieberman, Masaya Yoshida \& Colin Phillips. 2007. The effect of syntactic constraints on the processing of backwards anaphora. Journal of Memory and Language 56. 384-409.

Lewis, Richard \& Shravan Vasishth. 2005. An activation-based model of sentence processing as skilled memory retrieval. Cognitive Science 29. 1-45. http: //www.ling.uni-potsdam.de/ vasishth/Papers/Lewis-VasishthCogSci2005.pdf.

Moltmann, Friederike. 1992. Reciprocals and sameldifferent: Towards a semantic analysis. Linguistics and Philosophy 15(4). 411-462.

Radó, Janina \& Oliver Bott. to app. Underspecified representations of quantifier scope? In Maria Aloni, Vadim Kimmelman, Floris Roelofsen, Galit Weidman Sassoon, Katrin Schulz \& Matthijs Westera (eds.), Logic, language and meaning: 18th Amsterdam Colloquium, The Netherlands: Springer.

Reinhart, Tanya. 2006. Interface strategies: Optimal and costly computations. Cambridge, Massachusetts: MIT Press.

Roeper, Thomas, Barbara Z. Pearson \& Margaret Grace. 2011. Quantifier spreading is not distributive. In Nick Danis, Kate Mesh \& Hyunsuk Sung (eds.), BUCLD 35, 526-539. Somerville, MA: Cascadilla Press.

Trueswell, John, Michael Tanenhaus \& Susan Garnsey. 1994. Semantic influences on parsing: Use of thematic role information in syntactic ambiguity resolution. Journal of Memory and Language 33. 285-318.

Tunstall, Susanne. 1998. The interpretation of quantifiers: Semantics and processing. Amherst: University of Massachusetts dissertation.

Vasishth, Shravan \& Richard L. Lewis. 2006. Argument-head distance and processing complexity: Explaining both locality and antilocality effects. Language 82(4). 767-794. http://www.ling.uni-potsdam.de/ vasishth/pdfs/ Vasishth-Lewis-Language2006.pdf. 
The Online Interpretation of Sentence-Internal Same and Distributivity

Jakub Dotlačil

UCSC, Linguistics

1156 High St, Santa Cruz

CA 95064

j.dotlacil@gmail.com
Adrian Brasoveanu

UCSC, Linguistics

1156 High St, Santa Cruz

CA 95064

abrsvn@gmail.com 